Procesos especulares y refracción de los proyectos: Dos Proyectos de Título en las antípodas

Mirror processes and project refractions: uxtaposition of two graduate degree projects

Francisco Allard S.

Diego Rossel P.

Editado por: Diego Vallejos 0.

\title{
$<$ Resumen>
}

Dos proyectos de titulación de la generación 2004 (que desarrolló su Proyecto de Título durante 2005 ) son presentados para explorar los márgenes temáticos de este número de "De Arquitectura".

$<$ Abst ract $>$

The discussion of two graduate degree projects, focused on the topic of this issue of De Arquitectura.

<Palabras clave>

PROYECTO DE TÍTULO / PROCESO DE DISEÑO ARQUITECTÓNICO / ARQUITECTURA RELIGIOSA / ARQUITECTURA Y AUTOPISTA URBANAS

$\langle$ Key words >

GRADUATE DEGREE PRO] ECT / ARCHI TECTURE DESI GN PROCESS / RELI GI OUS ARCHI TECTURE / ARCHI TECTURE AND URBAN HI GHWAYS

\section{Introducción}

Durante el proceso de titulación 2005, los alumnos podían elegir profesor guía entre los siguientes arquitectos:

\section{Prof es or es guí as}

Para proyecto de Título:

Prof. José Camplá / Prof. Juan Cárdenas, Prof. Yves Besançon / Prof. Reginald Budd / Prof. Humberto Eliash / Prof. Andrés Elton / Prof. Pablo Gil / Prof. Pedro Gubbins / Prof. Jorge Iglesis / Prof. Patricio Morelli / Prof. Gustavo Munizaga / Prof. Oscar Ortega / Prof. Javier Pinto / Prof. Leopoldo Prat / Prof. Alberto Sartori / Prof. Edwin Weil.
Para tesis de Título:

Prof. Enrique Walker - Depto. de Diseño / Prof. María Bertrand - Depto. de Urbanismo.

Como marco referencial se les indicó lo siguiente:

Definición de tema y programa:

“ (...) Aún cuando el tema es de elección de los postulantes, la Escuela recomienda mantenerse dentro de las temáticas de interés nacional, concordantes con la misión de la Universidad de Chile, tales como: Vivienda Social, Reforma Educacional y Judicial, Equipamiento de Salud u otros temas urbanos de contingencia nacional. La elección del tema y formulación del programa es uno de 


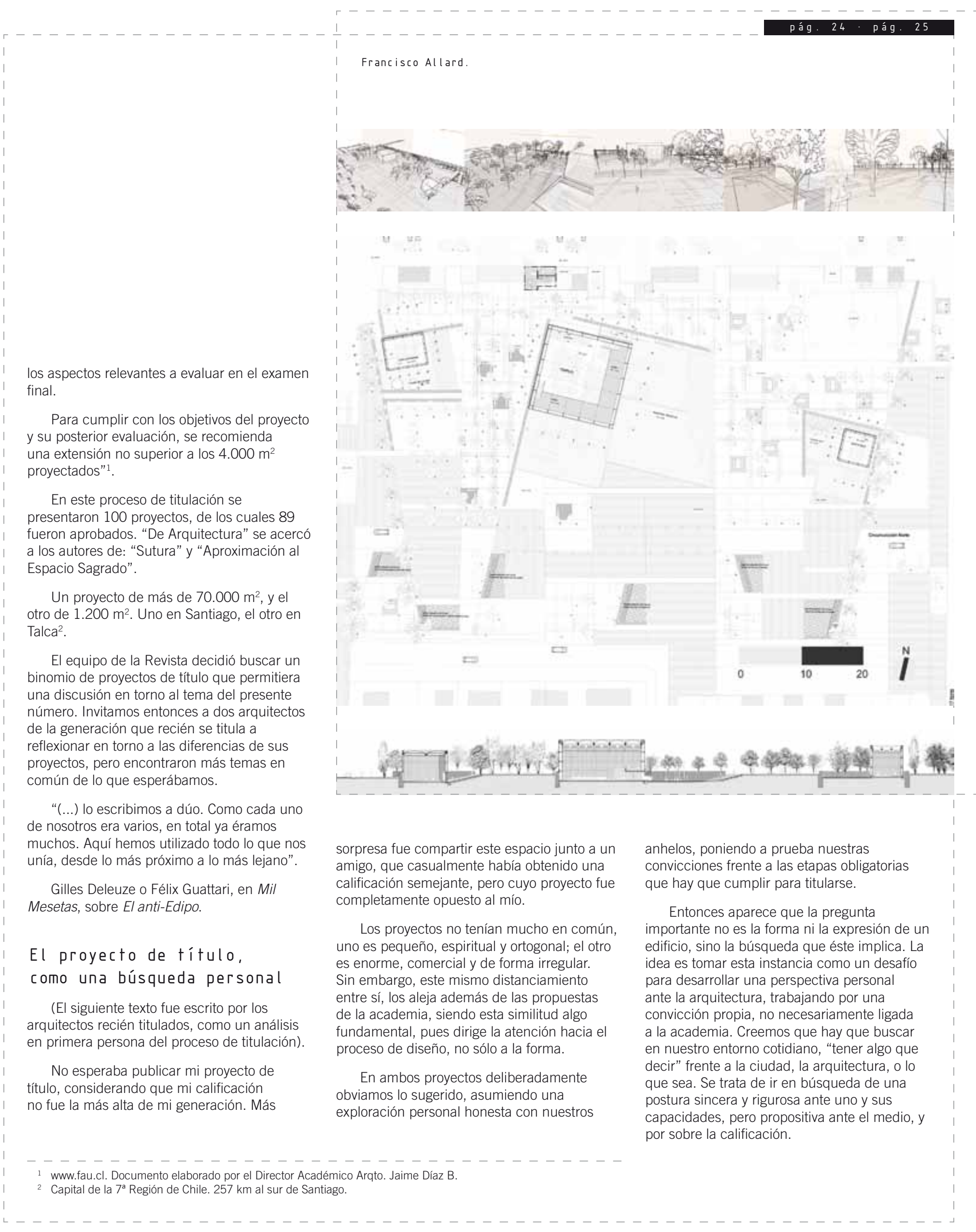




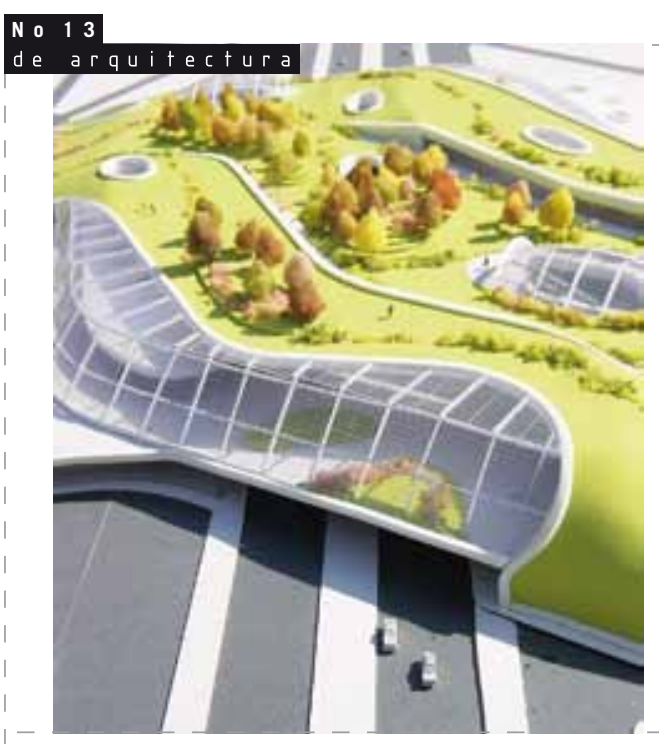

Esta tarea es difícil, pues siempre está el temor al prejuicio: "el proyecto de título es difícil, enorme, temible, angustioso e inevitable". Pero finalmente no es más que un proyecto de taller de $6^{\circ}$ año, con condiciones de entrega y correcciones similares a las de toda la carrera. Muchas veces este temor lleva a que los proyectos terminen siendo estrictamente correctos, sin generar nuevas propuestas o discusión sobre cómo enfrentar la realidad contemporánea.

Para proponer un discurso interesante, no es necesario hacer cosas exóticas, sino más bien buscar en lo cotidiano... en este caso: autopistas y capillas. El tema no nace de una exploración frenética por algo distinto o extravagante, más bien proviene de un universo muy cotidiano e íntimo, tan común que deja de ser algo presente. La investigación rescata aquello tan obvio que olvidamos, dándole un valor como tema de estudio.

Luego de tener claro qué decir, el resto es bien difícil, pero hay que tener convicción, perderle el miedo a lo que no se puede hacer. Si bien tuvimos problemas para obtener el pase $^{3}$, damos fe de que se pueden sacar adelante proyectos que no son repeticiones de modelos clásicos (el centro de... la escuela, el centro cultural de... etc.), son temas que no han sido aprobados previamente ${ }^{4}$, de hecho, no son temas que hayan sido realizados como ejercicios en el pregrado.

Los profesores guía no siempre se arriesgan, obviamente se prefieren proyectos preaprobados que cumplan con la normativa.
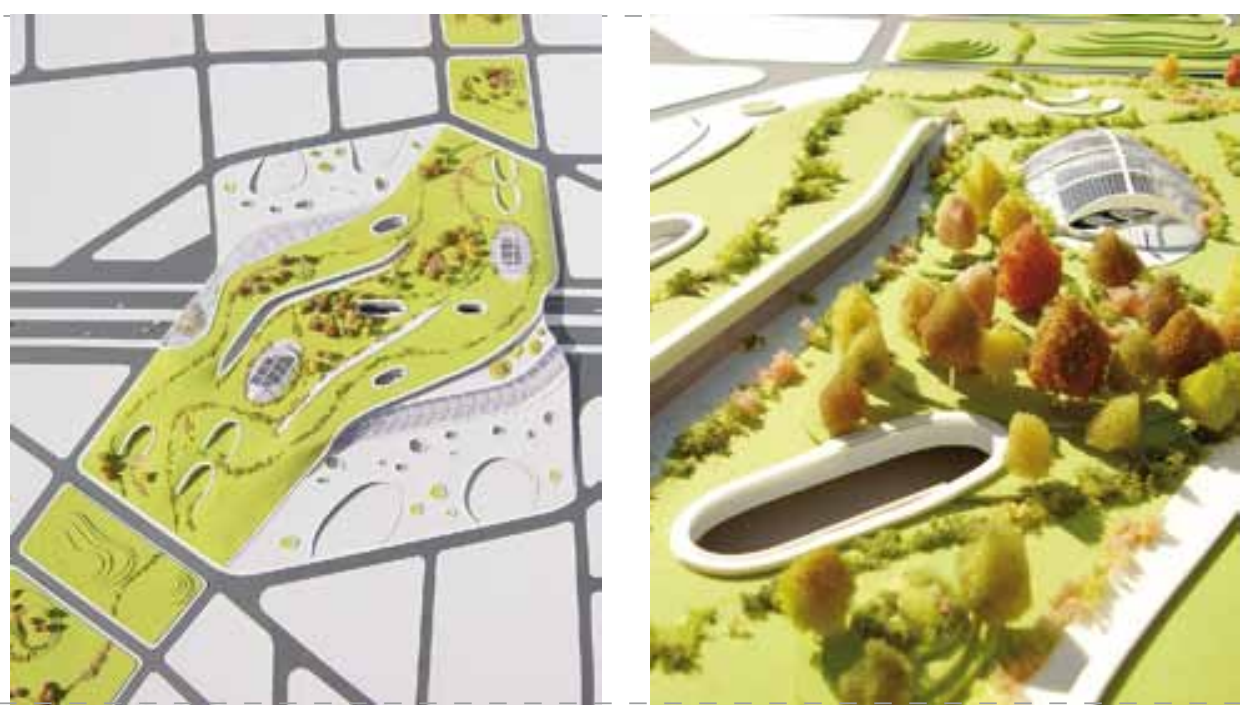

Entendemos que está en el espíritu de la "definición del tema..." por una parte: ahorrarle tiempo al alumno, para que empiece luego su trabajo; y por otra enfrentar temas reales de importancia para la sociedad, pero siempre definidos finalmente por los alumnos. Es esto último es lo que nos dio la opción de desarrollar nuestros proyectos.

\section{La calificación}

Los proyectos fueron calificados con nota 6.8 y 6.5 , lo cual nos indica que si bien son proyectos "correctos", tienen una distancia al canon de la academia. Creemos que el proyecto de título, es un punto de quiebre con la academia. Quienes han llegado a la instancia de Título ya han demostrado sus habilidades de estudiante de arquitectura, son todos Licenciados en Arquitectura. Entonces ¿Es la primera instancia profesional? o ¿La última instancia académica?, a nosotros se nos apareció como una oportunidad de quiebre: de, al amparo de la institución, elaborar un discurso propio. En este marco, parece que la calificación nos indica que no estábamos tan equivocados.

\section{Algunas conclusiones}

La temática dialéctica original de: grande versus pequeño, no resulta ser una metodología suficiente para definir la arquitectura. La "escala" es un tema muy importante, como se verá a continuación. Los proyectos no se explican en su tamaño, sino en su relación con el entorno y principalmente con su proceso de producción.

"Sutura", un proyecto metropolitano, no logra una resolución detallada de su arquitectura. No es posible detallar todo el proyecto, son los criterios estructurales y de partido general los que lo explican.

"Aproximación al Espacio Sagrado", una acción local, alcanza a mostrar los detalles constructivos del proyecto. Hasta la selección de los árboles es parte del discurso arquitectónico.

La selección de estos dos proyectos de título, finalmente sirvió para explorar la temática misma del proceso de titulación. Los procesos fueron muy semejantes, y quizás la diferencia en la forma es sólo una refracción posible dentro del esquema que ofrece la escuela. Tal vez si hubiera más tiempo ${ }^{5}$, si se contara con más recursos económicos ${ }^{6}$ o si la escuela planteara líneas de investigación claras ${ }^{7}$, los proyectos podrían llegar a compararse formalmente. Finalmente son los procesos los evaluados, la forma es sólo una de sus manifestaciones. Quizás este sea un valor de la Facultad de Arquitectura y Urbanismo (FAU), dar cabida a intereses personales en un ámbito profesionalizante. Esto permite explorar ideas profesionales desde la academia, sin las limitaciones de la realidad. Por eso encontramos importante aprovechar esta instancia para probar, no sólo aprobar.

\footnotetext{
Cuando el Profesor Guía está de acuerdo con el avance del proyecto, en una última corrección, da "El Pase" luego del cual el alumno debe desarrollar "la entrega" del proyecto. Hay proyectos similares, pero no son comunes.

Actualmente todos los proyectos, sin excepción, tienen un año para terminarse. Si alguien no alcanza, debe empezar un nuevo proyecto, con nuevo profesor guía, tema y terreno.

Si bien durante el $6^{\circ}$ año se libera al alumno del pago del arancel, eso no implica disponer de ese dinero para el desarrollo del proyecto.

Ver: "Definición del Tema". Finalmente no se plantea una línea aparte de proponer arquitectura pública.
} 


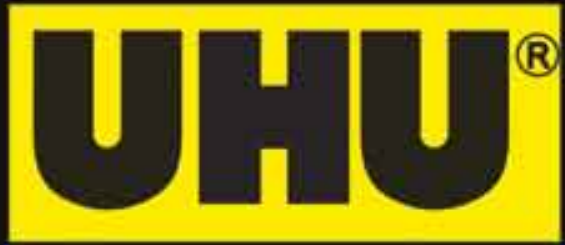

MADE IN GERMANY

Adhesivos para múltiples usos:

[UHU Papeleria / "UHU-Tac"] Masilla adhesiva removible: Fácil de usar, práctico, rápido y limpio. Sirve para fijar afiches, carteles ligeros o pequeños objetos en superficies lisas. No deteriora paredes. Reutilización múltiple.

\section{[UHU Hogar / Frasco Fácil]}

Pegamento universal transparente en un práctico envase con aplicador adaptable.

Para pegados por punto, por linea y por área.

[Pegamento en Barra "UHU stic"] Rápido, limpio, fácilmente lavable, no tóxico.

Con tapa rosca que evita que se seque.

\section{[Pegamento Universal]}

Fácil de usar y útil en prácticamente todo tipo de materiales. Cristalino y limpio.

¡Pegar es amarillo!
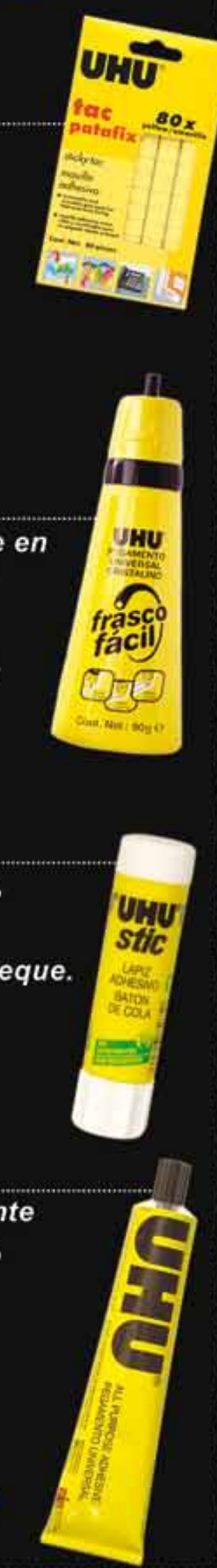

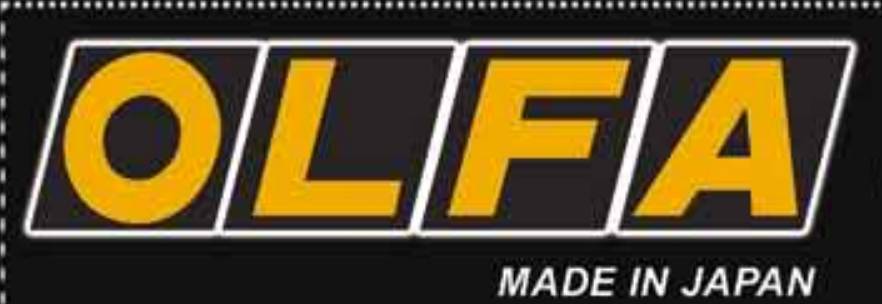

(B)

\section{¡El mejor fabricante de herramientas cortantes del mundo!}

[Cuchilla Artistica AK-4] Útil para trabajos gráficos, artísticos y de precisión.

[Cuchilla Corte $45^{\circ} \mathrm{MC}-45$ ] Facilita cortar esquinas de $45^{\circ}$, por ejemplo, cortes de fotografias.

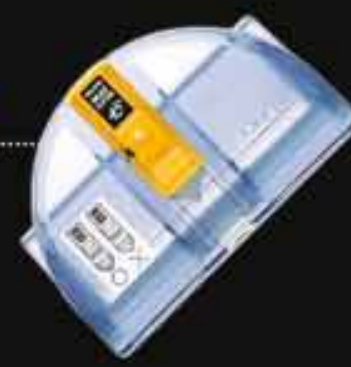

[Cuchilla para Acrilicos P-800]

Corta diferentes tipos de plásticos, acrilicos y laminados.

[Cuchilla Gancho HOK-1] Corta cintas plásticas sin dañar el contenido de las cajas.

\section{[Cuchilla Compás CMP-1/DX]} Cuchillo giratorio, especial para cortes de circulos en papeles y láminas.

\section{[Cuchilla Rotativa RTY - 2/G]}

Primer cortante de cuchilla circular, corta gran cantidad de materiales. Apta para diestros y zurdos.

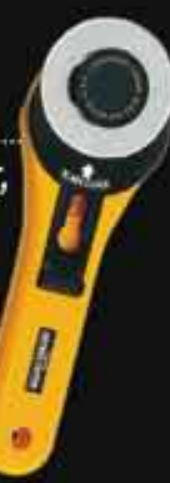

[Cuchilla Rotativa PRC-2]

Utilizado especificamente para hacer cupones, diseñar tarjetas y prepicar hojas.

\section{jCortar es Olfa!}


Aproximación al Espacio Sagrado

Francisco Allard

Profesor guía: Pablo Gil

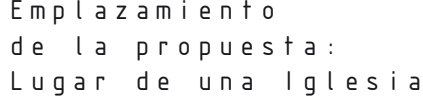

"El templo católico -esto es la iglesia-es el espacio arquitectónico en que se reúne la comunidad de fieles, es la casa del pueblo de Dios. Casa de Dios, en cuanto a que la misma asamblea de los fieles constituye el templo del Dios Vivo".

fr. José Manuel de Aguilar ambientes arquitectónicos para la administración de los sacramentos. En: arte sacro Concilio Vaticano Segundo, pág. 187.

El principal motor que define el lugar sagrado es una comunidad concreta que está en busca de una iglesia.

T a l c a

Con una población de (189.505 hab.), que aún conserva tradiciones y mentalidad del mundo agrícola, apreciable en el modo de vida, en sus costumbres, tradiciones y en sus celebraciones.

Un porcentaje cercano al 70\% de la población se declara católico, población que tiene las intenciones de buscar a un Dios que se entienda bajo la fe cristiana.

La comunidad a trabajar en el proyecto es de 2.773 familias, ubicadas en la nueva zona de expansión de la ciudad, Talca, definida por los límites, al norte por un paño agrícola, al oeste por la línea férrea, y al este por la carretera.

$$
\begin{aligned}
& \text { Nuevo escenario: } \\
& \text { Plano regulador }
\end{aligned}
$$

Deja la zona delimitada por la nueva circunvalación metropolitana, conectándola al resto de la urbe y generando una nueva situación de borde. Cambia el uso de suelo de predio agrícola a suelo de densidad habitacional media (AU6), correspondiente a una población aproximada de 9.000 familias.

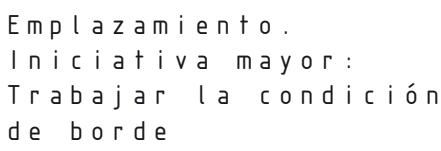

A través de un parque que recoge la dimensión completa del sector, trabajando el carácter de borde urbano-rural en un comienzo, generando una instancia de amortiguación y fusión de estas dos realidades, para luego tras ser urbanizada la zona agrícola

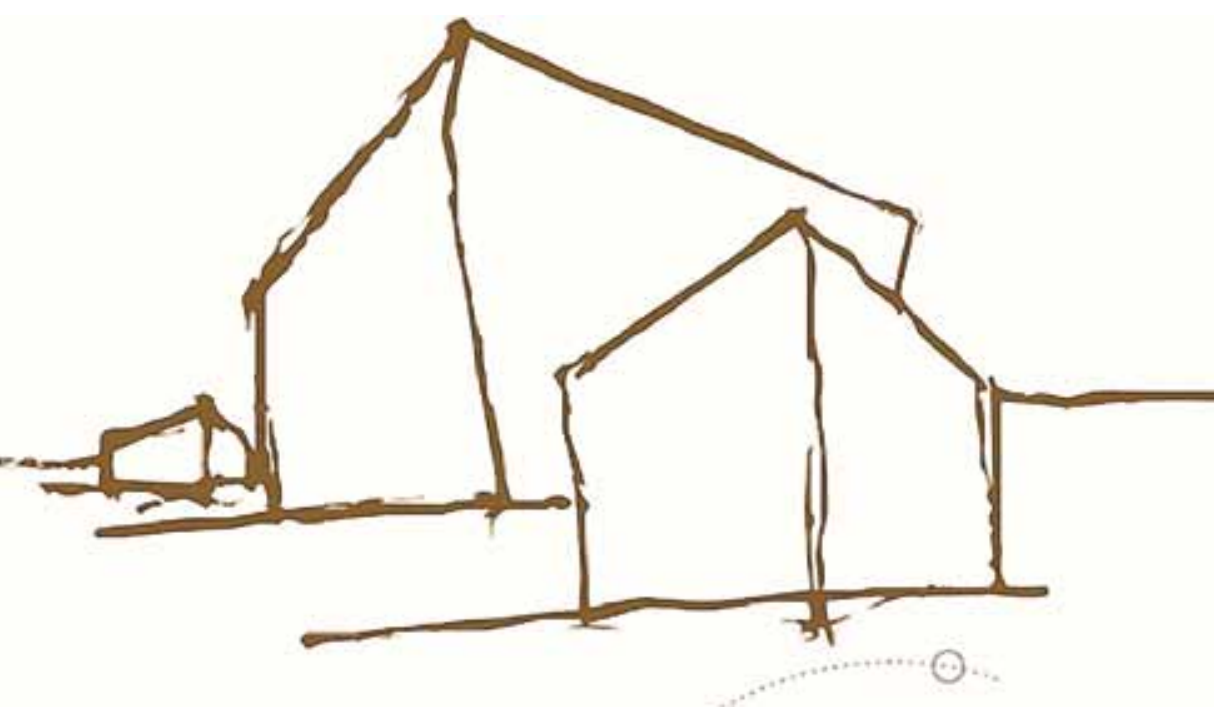

Iglesia: Relicario de un gran tesoro -sacramentos y liturgia- punto de relación vivenci con Dios, cofre que se abre para recibir a la comunidad. Cubo en su absoluta abstracción. búsqueda por la unidad y la centralidad, la quietud. Tres tesoros, representando os sacramentos de iniciación; vida y muerte, como parte de una cosmovisión mayor:

del significado de la existencia.

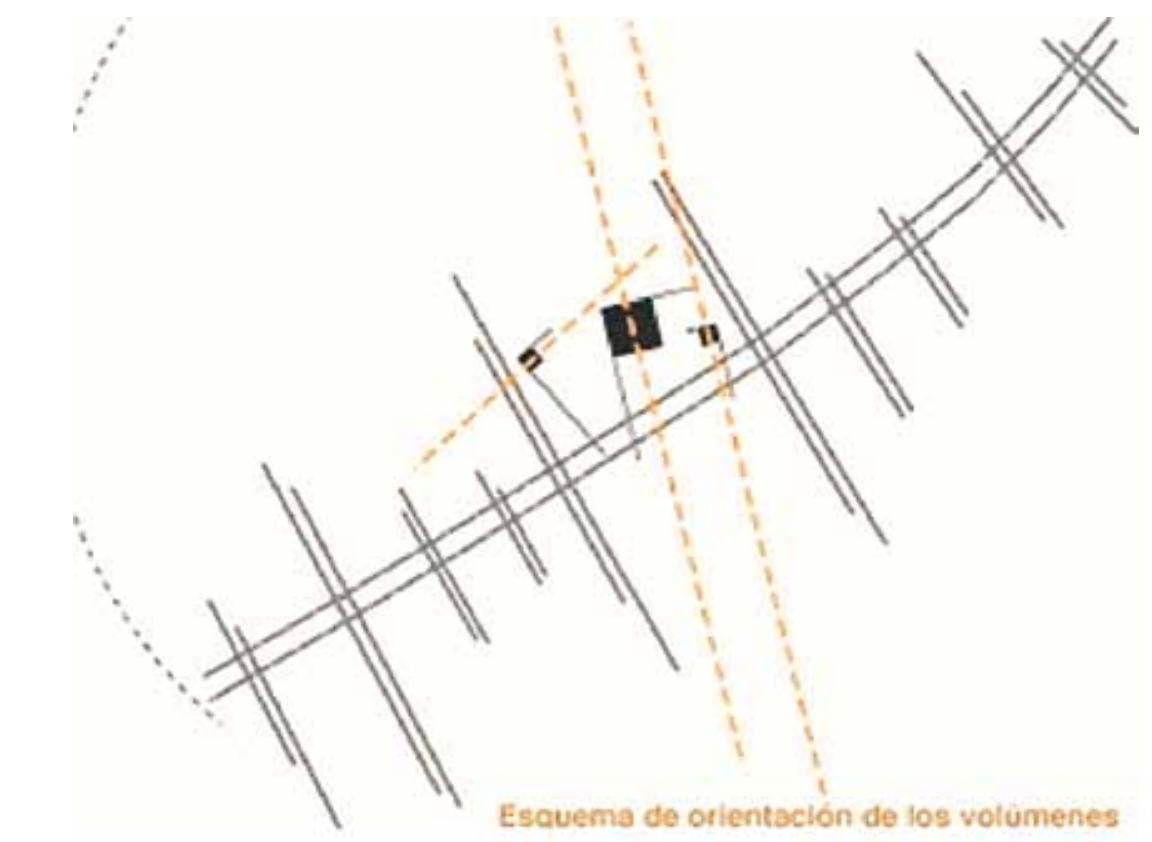

convertirse en el testigo silencioso de un pasado rural y a la vez en el articulador de la realidad existente con la que llegará

Dualidad naturaleza -ciudad como una equivalencia entre lo divino y lo humano-.

$$
\begin{aligned}
& \text { La iglesia como la casa } \\
& \text { de Dios y de los hombres }
\end{aligned}
$$

Orientación, hacia el oriente como condición de vida y esperanza. Hacia el poniente, carácter final, ocaso de la vida terrestre y paso a un nuevo estado.

Doble Postura: Carácter individual y divino, y a su vez, el carácter mundano y cotidiano; son difíciles de conciliar y fáciles de reconocer.
Así aparece la imagen de Jesucristo como nexo entre estos dos mundos:

- Naturaleza - Divino

- Sobrenatural - Humano

- Dios - Hombre

- Redentor - Carpintero.

Iglesia al igual que Jesús: Humana en su exterior, pero divina en su interior.

Madera exterior: Símbolo del trabajo del hombre.

Luz interior: Condición atmosférica que nos habla de la presencia divina del Espíritu Santo. 

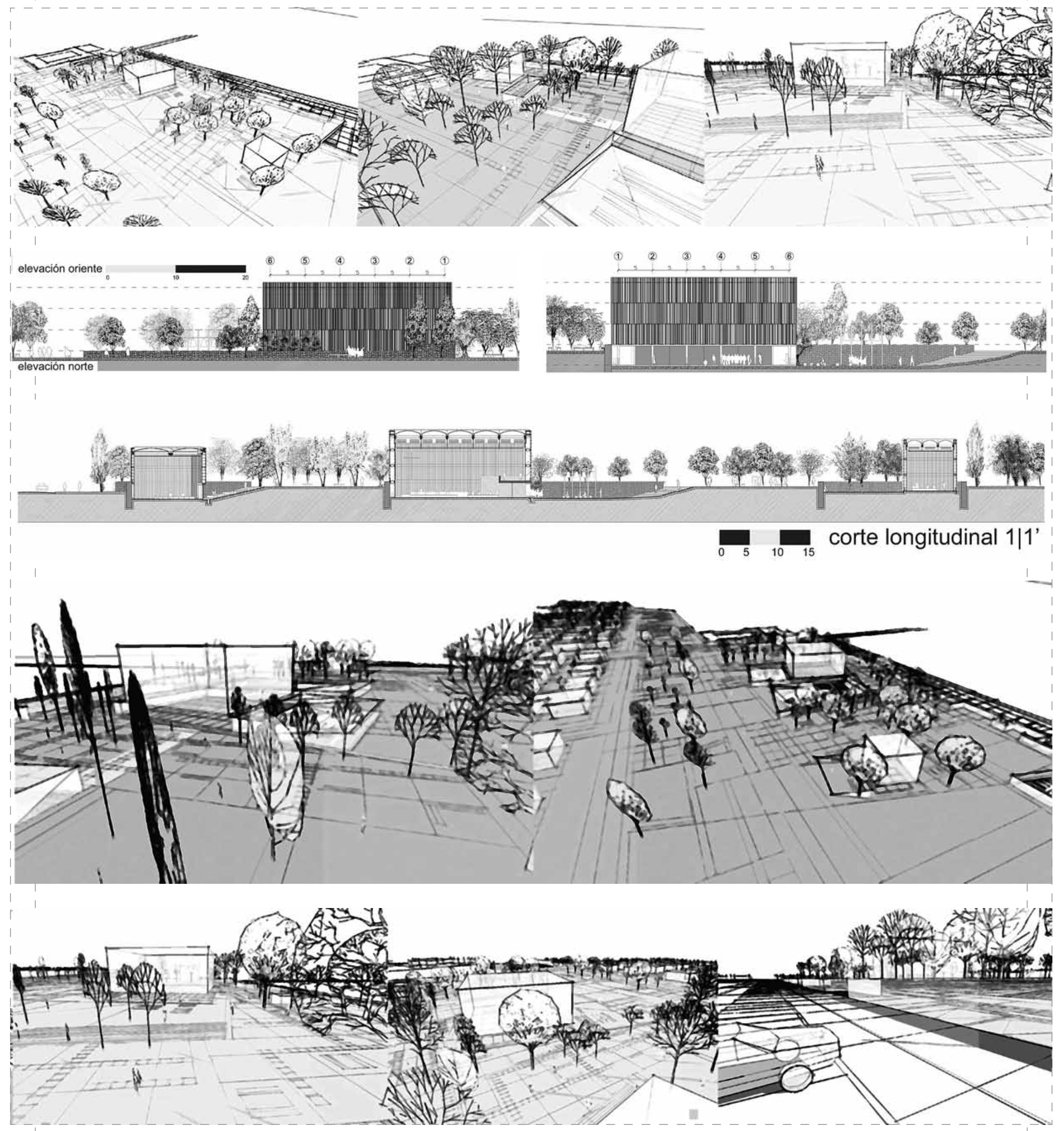


\section{Sutura}

Diego Rossel

Profesor Guía: Pablo Gil

$$
\text { objetivo }
$$

Hacerse cargo de la mejor manera posible del problema de segregación provocado por la existencia de las autopistas, teniendo conciencia de que los actuales sistemas de conexión (pasarelas peatonales) no logran resolver los problemas provocados por ellas.

Explorar nuevas posibilidades de dar continuidad a los tramos de ciudad divididos por la autopista, no sólo conectando puntos, sino que a través de una integración programática.
Constituir o colonizar estos espacios como lugares y hacerlos parte de la ciudad.

$$
\text { Propuestaconceptual }
$$

De acuerdo a los objetivos y al conocimiento de las actuales estructuras que dan una supuesta solución al problema de segregación causada por la autopista, la propuesta consiste en dar continuidad al tejido urbano, mediante la reformulación de la pasarela.

La pasarela debe perder su condición de objeto, entendiéndose entonces como una continuación de la trama urbana, la cual deberá incorporar una mayor cantidad de factores que hacen ciudad, sean estos: funcionales, formales, espaciales, emocionales, culturales y principalmente programáticos con la intención de restituir en parte las relaciones que hubo entre un borde y otro actualmente quebrantadas por la intromisión de la autopista.

Para propiciar estas situaciones surge como primera premisa de diseño la necesidad de aumentar la dimensión del soporte que permite al peatón transitar sobre la autopista.

En síntesis: "Continuar el plano de la ciudad" a través de un espacio que entregue a los peatones la mayor cantidad de posibilidades, ya sea en cuanto a decisiones de movimiento, de acciones y de apropiación, aumentando la superficie de conexión para lograr así un espacio que amplíe las posibilidades de interacción.

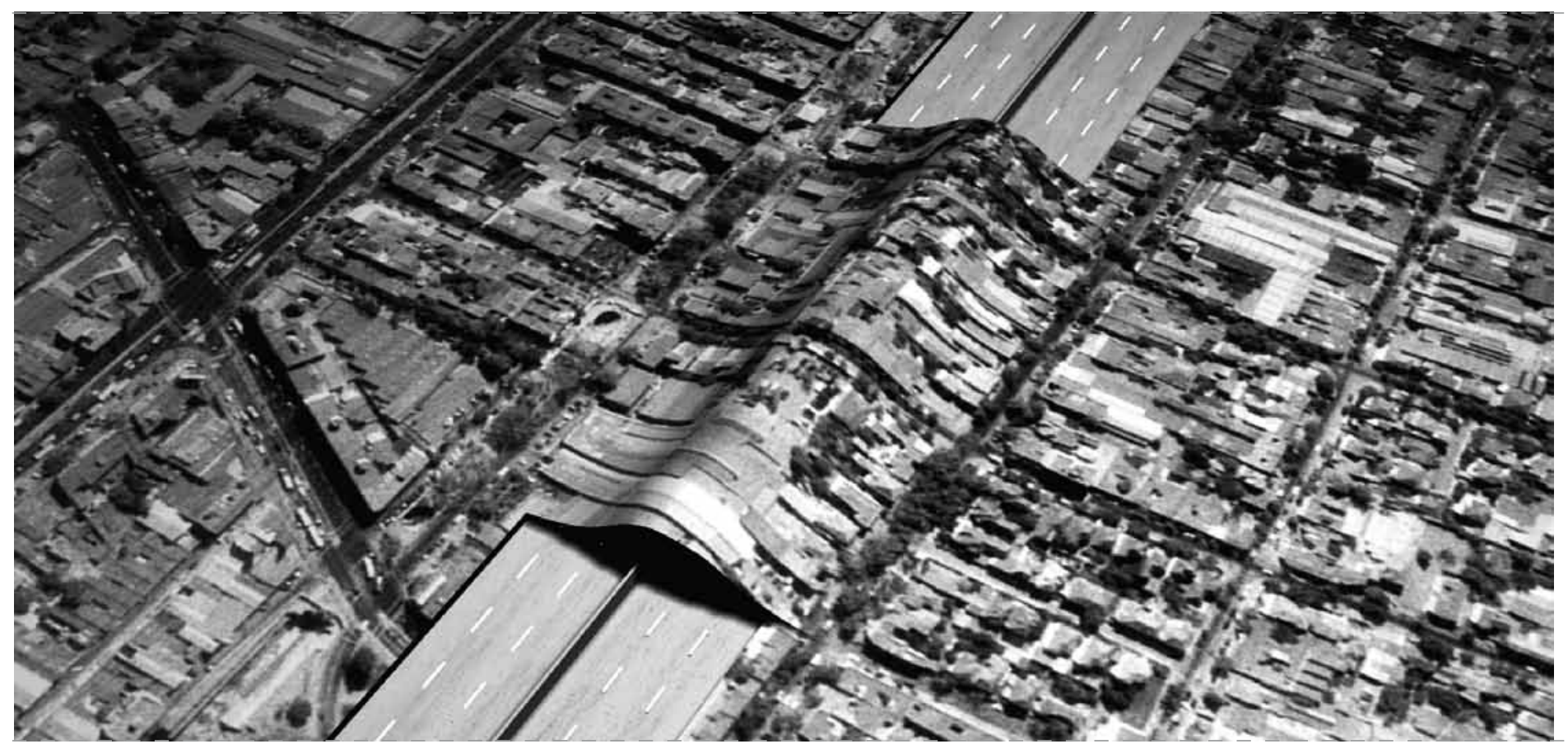

¿Cómo continuar el plano de l a c i udad?

1. Constituyendo la pasarela más que como un "paso", como un "lugar de paso".

2. Mediante el construir que significa cuidar, dar protección, preservar de la amenaza, cuidar de los daños, para así poder habitar el lugar, a través de actividades y programas.

3. Afectar este espacio a través de los bordes (importancia de la proximidad de los bordes y de que existan actividades en ellos).
4. Más allá de lograr una continuidad física lo que se busca es generar una continuidad programática.

\section{¿Cómo?}

Esto no sería posible sin la presencia de un programa capaz de sustentarlo. Es por eso que de acuerdo a los preceptos y protocolos de diseño anteriormente mencionados, el programa más adecuado para estos efectos es el Mall.

$$
\text { ¿Por qué un Mall? }
$$

El mall se presenta entonces como un programa lo suficientemente seductor para el nuevo habitante metropolitano (automovilista) y con una oferta lo suficientemente variada para satisfacer las necesidades de consumo, servicios, recreación y equipamiento de los habitantes de ambas comunas, en especial de Pedro Aguirre Cerda.

Además tiene una escala lo

suficientemente grande como para otorgar un apropiado soporte al parque dispuesto en su superficie.

Estructuralmente el Mall y en general aquellos proyectos comerciales que tienen una pilarización regular, están diseñados para soportar grandes cargas verticales y resistir óptimamente a los esfuerzos sísmicos. 

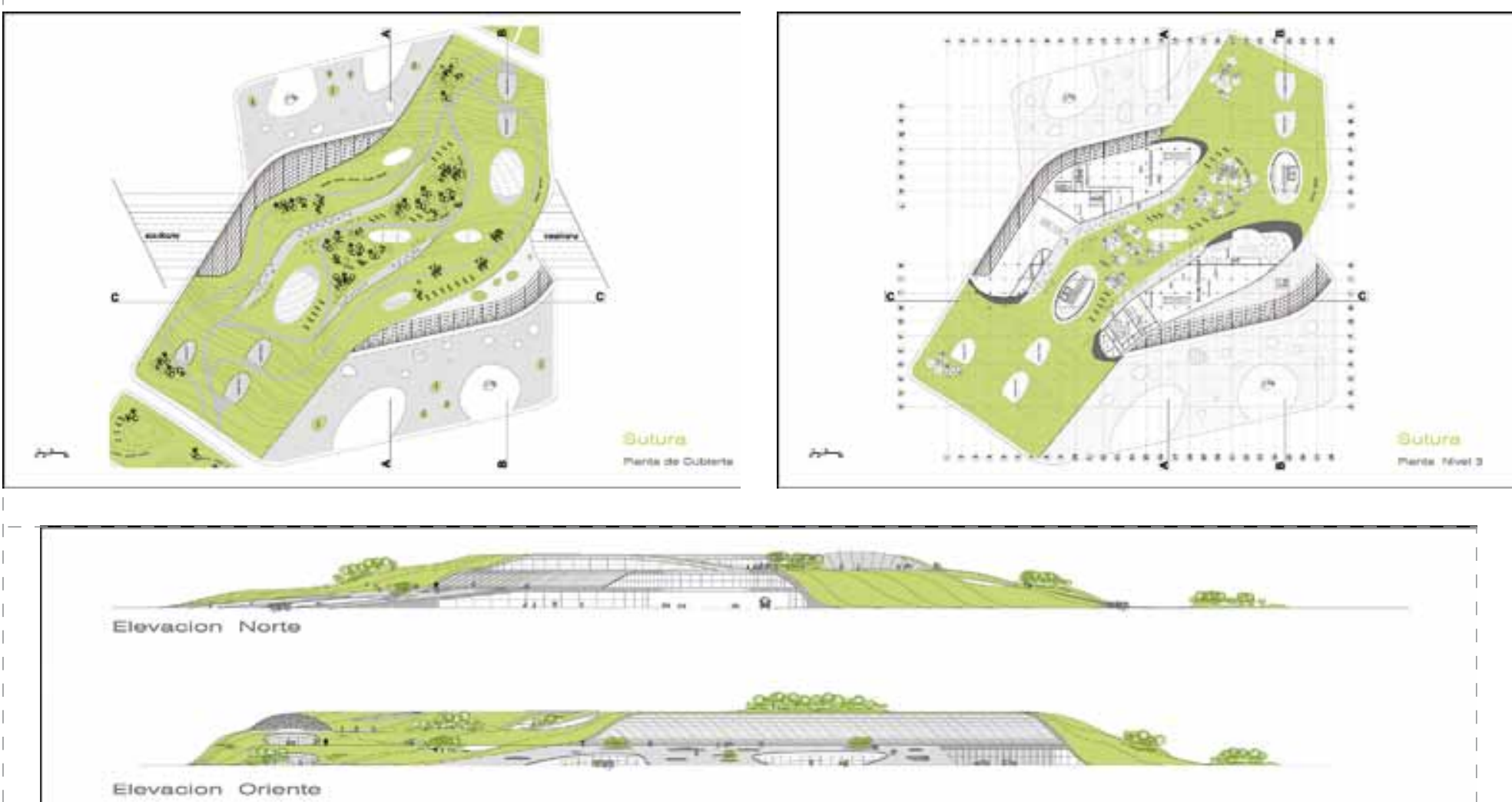

Elovacion Oriente

Elovacion Poniente
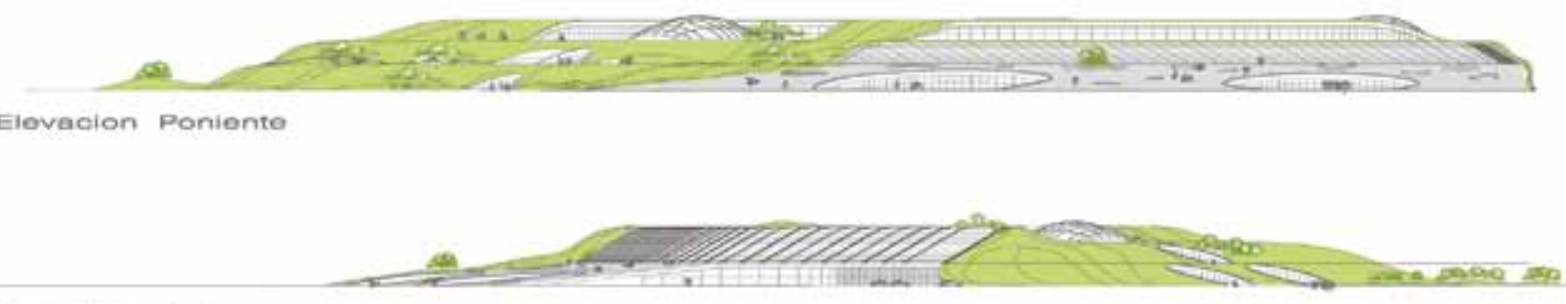

Elevacion Sur

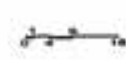

Sutura

Elovaciones
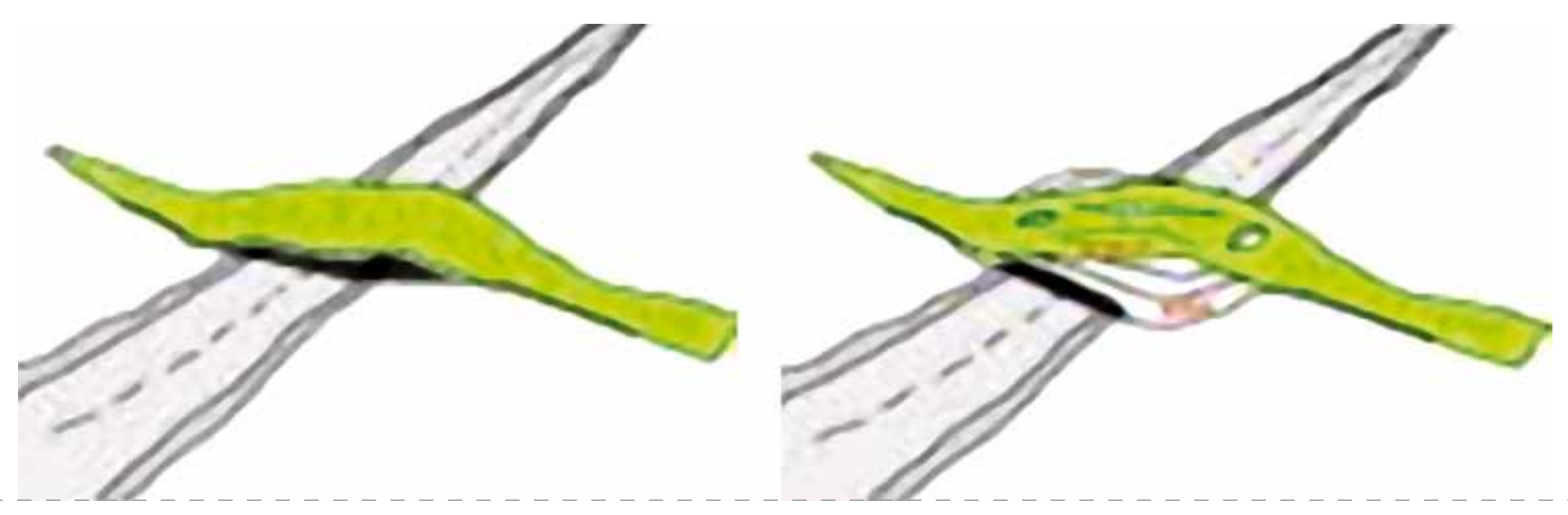